\title{
Ambulance Use by International Travelers in Japan: A Retrospective Descriptive Study
}

\author{
Yusuke Oshita $^{1 *}$, Koki Tsuchiya ${ }^{1}$, Koji Ishikawa ${ }^{1}, K^{\prime}$ Kodai Hirabayashi ${ }^{1}$, Tetsuya Nemoto ${ }^{1}$ \\ ${ }^{1}$ Department of Orthopaedics Surgery, Yamanashi Red Cross Hospital, Yamanashi, Japan
}

Corresponding Author: Yusuke Oshita, MD, PhD, Director, Department of Orthopaedics Surgery, Yamanashi Red Cross Hospital, Yamanashi, Japan. Tel: +81-0555-72-2222, Fax: +81-0555-73-1385, Email: oshita@med.showa-u.ac.jp

Received November 3, 2019; Accepted February 27, 2020; Online Published March 11, 2020

\begin{abstract}
Introduction: Reports indicate that $22 \%-64 \%$ of travelers experience some illness when in a foreign country. To date, no prior study has reported the use of ambulances by travelers or the epidemiology of travel-related injury.

Methods: In this retrospective study, we aimed to describe ambulance use by international travelers, including the rates of travel-related injury and illness. To do so, ambulance dispatch data from January 1, 2010 to December 31, 2018 was used.

Results: Overall, of the 43201 cases of ambulance use during the study period, 524 (1.2\%) were international travelers. Ambulance use by international travelers increased from $0.35 \%$ in 2010 (15/4311) to 2.54\% in 2018 (125/4913), an average annual increase of $0.27 \%$. Of the international travelers, $392(74.8 \%)$ had minor complaints, 110 cases (21.0\%) had moderate complaints, $280(53.4 \%)$ had internal disease, and 223 cases (42.6\%) had suffered trauma. Regarding location, 253 (48.3\%) were from a hotel/lodge, 83 (15.8\%) were from a $\mathrm{road} /$ parking, and $30(5.7 \%)$ were in the forest/mountain.

Conclusion: Most international travelers use ambulances for minor complaints, typically internal disease or trauma, and approximately half access the service from a hotel or lodge.

Keywords: Ambulance, Travel Medicine, Emergency, Trauma, Injury, Japan
\end{abstract}

Citation: Oshita Y, Tsuchiya K, Ishikawa K, Hirabayashi K, Nemoto T. Ambulance use by international travelers in Japan: a retrospective descriptive study. Int J Travel Med Glob Health. 2020;8(1):13-17. doi:10.34172/ijtmgh.2020.02.

\section{Introduction}

It is difficult for international travelers to obtain reliable medical information in the event of an emergency, which is compounded by differences in healthcare systems between countries. In Japan, the ambulance system is accessed to transport patients immediately to the nearest hospital or clinic. Although this is indispensable in preventing fatalities, it can fail to allow the assessment of the severity of a patient's status. International travel is increasing worldwide, and some travelers will become ill or injured and call an ambulance. However, how many cases are involved or what disease need are, specifically in Japan, is not known. To date, unlike travel-related illness, ${ }^{1}$ injury has not been well reported in previous research.

The current study aimed to report on ambulance use by international travelers and describe the characteristics of travel-related injury and illness in Japan.

\section{Methods}

This retrospective study was conducted from January
1, 2010 to December 31, 2018 after institutional review board approval was received from the Yamanashi Red Cross Hospital. Anonymized ambulance dispatch data was retrieved from the Fujigoko Fire Department (6-2-6 Shimoyoshida, Fujiyoshida-shi, Yamanashi, Japan), which services the northern and eastern sides of Mount Fuji. From this sample, the following data about travelers was collected based on the address information recorded by the dispatch center, which was independent from this study: age, gender, time of emergency request, place of emergency request, and disease and severity. Severity was classified according by the first assigned doctor as minor (can walk and no need for admission), moderate (cannot walk and needs admission), or severe (life-threatening condition, including CPA). No statistical analysis was performed in this study, and only descriptive data is presented (i.e. numbers and percentages).

\section{Results}

Overall, there was an upward trend in the percentage of international travelers requiring ambulance dispatch

Copyright $\odot 2020$ The Author(s). This is an open-access article distributed under the terms of the Creative Commons Attribution License (http:// creativecommons.org/licenses/by/4.0), which permits unrestricted use, distribution, and reproduction in any medium, provided the original work is properly cited. 
between 2010 and 2018 (Figure 1). In 2010, international travelers accounted for 15 of the 4311 ambulance dispatches (0.35\%), which increased annually to 125 of 4913 (2.54\%) in 2018 (Figure 1).

Among the cohort of 524 international travelers who used ambulance services during the study period, 220 were males and 304 were females, and the mean \pm SD age was $40.2 \pm 21.3$ years (range: 1-85 years). In order of decreasing frequency, the travelers originated from China $(n=242 ; 46.2 \%)$, Taiwan $(\mathrm{n}=56 ; 10.7 \%)$, Thailand $(\mathrm{n}=56 ; 7.8 \%)$, USA $(\mathrm{n}=29 ; 5.5 \%)$, Australia $(\mathrm{n}=20 ; 3.8 \%)$, Hong Kong $(\mathrm{n}=15 ; 2.9 \%)$, Malaysia $(n=13 ; 2.5 \%)$, South Korea $(n=13 ; 2.5 \%)$, Indonesia $(n=11$; $2.1 \%)$, Singapore, France, and Vietnam $(n=9 ; 1.7 \%)$, Canada, Spain, and Philippines $(n=6 ; 1.1 \%)$, Russia $(n=5 ; 1.0 \%)$, Netherlands, Germany, and New Zealand ( $n=4 ; 0.8 \%)$, United Kingdom $(\mathrm{n}=3 ; 0.6 \%)$, Israel and Mexico $(\mathrm{n}=2$; 0.4\%), Ireland, Afghanistan, Italy, Cambodia, Saudi Arabia, Switzerland, Slovenia, Czech Republic, Denmark, Bhutan, Brazil, Belgium, and Myanmar $(\mathrm{n}=1 ; 0.2 \%)$, and unknown $(n=2 ; 0.4 \%)$. Figure 2 shows the distribution of these cases by month, with peaks evident during July and August. Figure 3 shows the distribution by time of day, with peaks seen around noon and in the early evening. The dispatch location was to a hotel or lodge 253 times (48.3\%), a road 51 times
(9.7\%), a parking facility 32 times (6.1\%), the forest/mountain 30 times (2.9\%), a restaurant 15 times $(2.9 \%)$, the market 15 times (2.9\%), to a station 14 times (2.7\%), and to other places 114 times (21.8\%).

Disease severity was rated as minor in 392 cases $(74.8 \%)$, moderate in 110 cases $(21.0 \%)$, or severe in 15 cases $(2.9 \%)$ with another 6 cases experiencing cardiopulmonary arrest (CPA) (1.1\%) and 1 case having an unknown severity (0.2\%). Concerning the diagnoses of patients, 280 cases $(53.4 \%)$ had internal disease, 223 (42.6\%) had trauma, 10 (1.9\%) had gynecological disease, 6 (1.1\%) had CPA, 1 (0.2\%) had dental disease, $1(0.2 \%)$ had mental disease, and $3(0.6 \%)$ were unidentified due to insufficient data. In the internal medicine subgroup, abdominal disease (24.6\%) and central nervous system disease (23.6\%) were most common (Figure 4). In the trauma subgroup, head, neck, and limb injuries were most common (Figure 5), and patients were typically diagnosed with contusion/wound (61.9\%) or fracture/ dislocation (31.8\%) (Figure 6). Of the 10 gynecological cases, services experienced 4 cases of threatened abortion, 3 cases of abortion, 2 cases of genital bleeding, and 1 case of a Bartholin's cyst. The most common requests for ambulance dispatch were from a hotel or lodge, and most of these calls were made in the evening or at night (Figure 7).
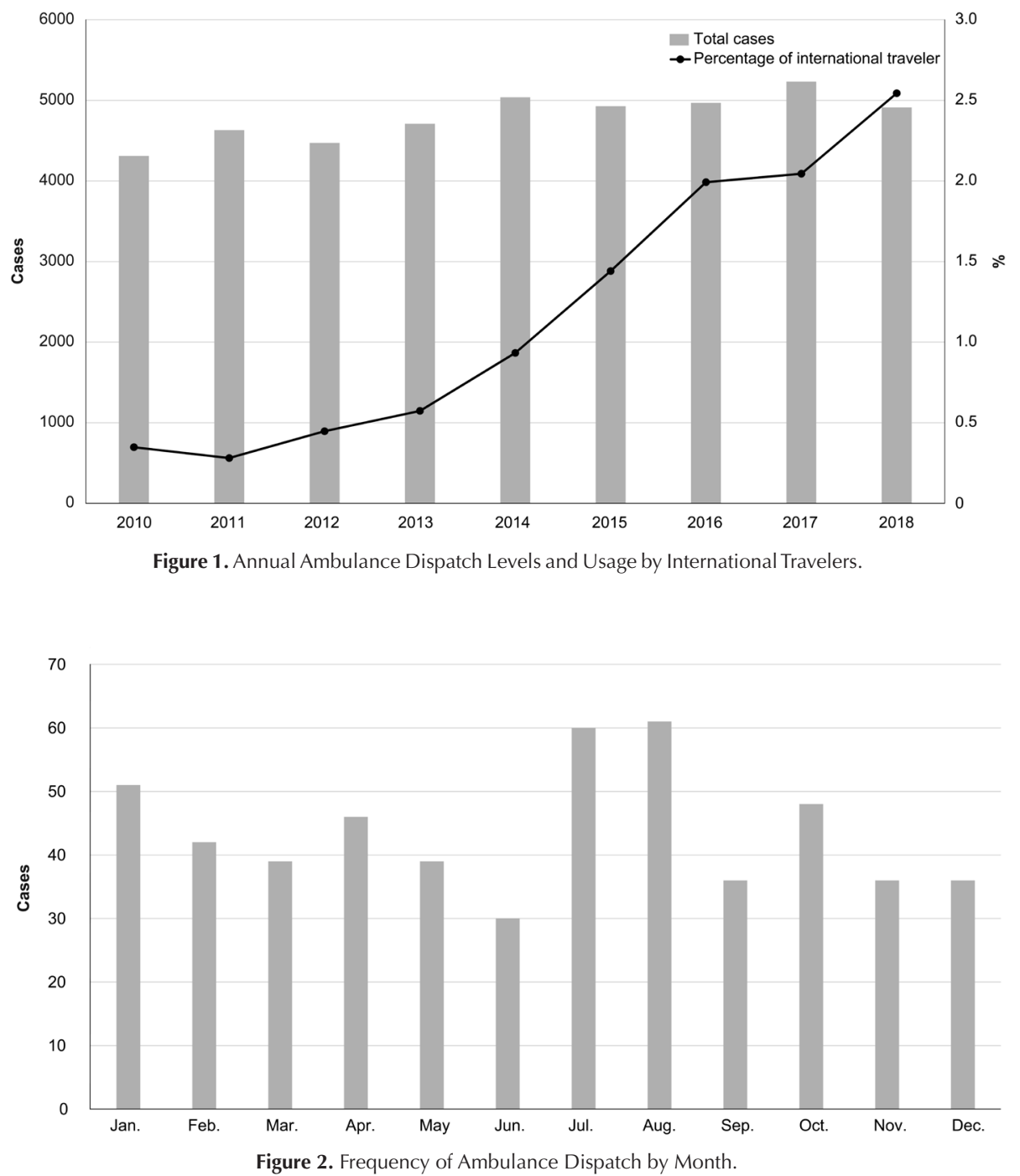


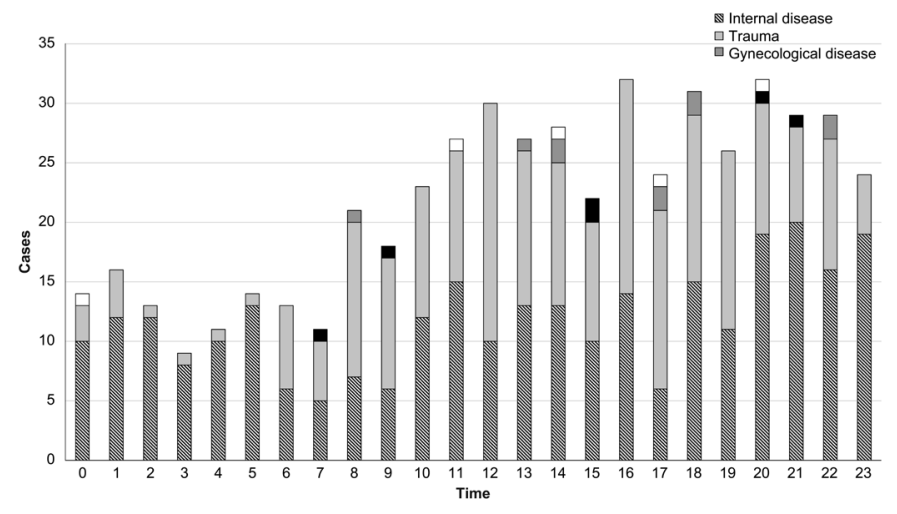

Figure 3. Frequency of Ambulance Dispatch by Time.

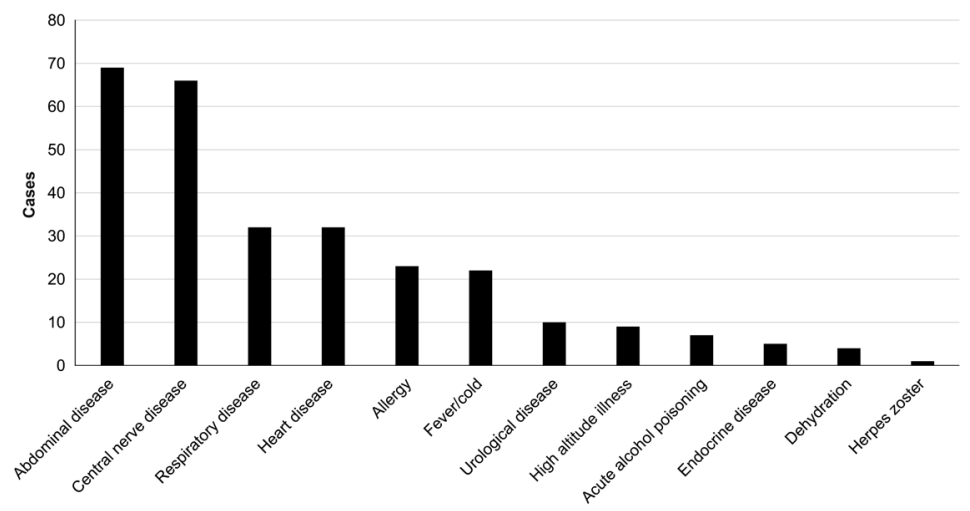

Figure 4. Diseases Presenting in the Internal Medicine Subgroup.

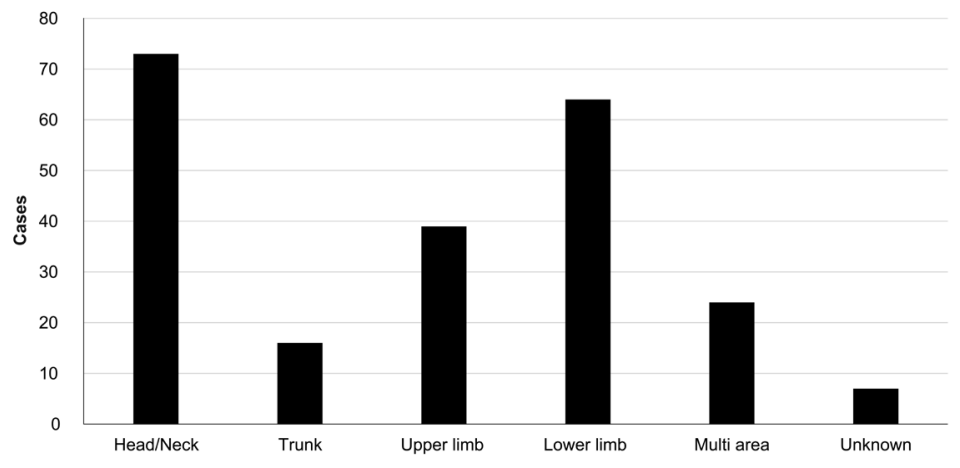

Figure 5. Injury Locations in the Trauma Subgroup.

\section{Discussion}

To the best of our knowledge, this is the first report to focus on ambulance use by international travelers in Japan. It is known that the main health problems among international travelers are diarrhea, ${ }^{2}$ cough, runny nose, sore throat, ${ }^{3}$ and activity-related problems. ${ }^{4}$ Fatal trauma is also reported, ${ }^{5}$ but cases of minor trauma are not well reported, meaning that key information about the demands of travelers on ambulance services for transport to a hospital or clinic is lacking.

The Japan National Tourism Organization ${ }^{6}$ reported that the number of foreign travelers to Japan has been increasing since 2011. Levels were 8611175 in 2010, fell to 6218752 in 2011, but have increased year after year thereafter to peak at 31191856 in 2018 (8358 105 in 2012; 10363904 in 2013; 13413467 in 2014; 19737409 in 2015; 24039700 in 2016; 28691073 in 2017). This represents an annual increase of approximately eight times (15-125). Corresponding with this, the rate of ambulance usage by international travelers also increased from $0.35 \%$ in 2010 to $2.54 \%$ in 2018 , an annual increase of $0.27 \%$. It is reported that $22 \%-64 \%$ of travelers report some illness, ${ }^{1,7}$ with most cases deemed mild and self-limiting, such as diarrhea, respiratory infections, and skin disorders. ${ }^{4}$ In 2016, the Ministry of Health, Labor, 


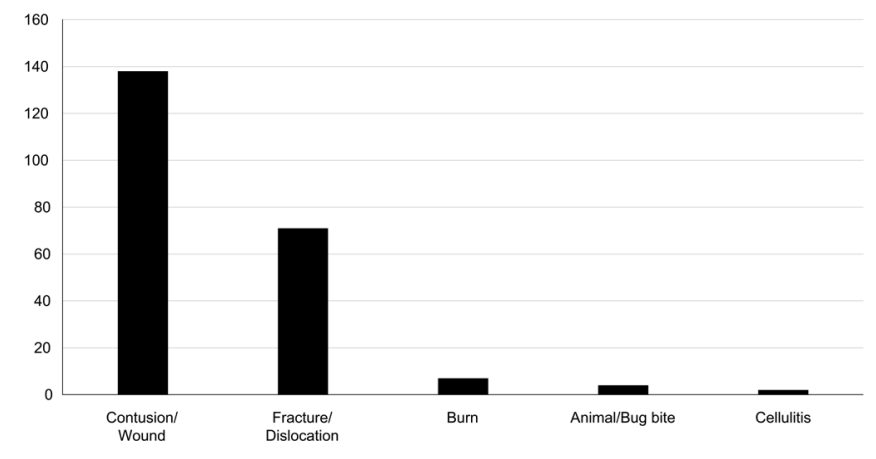

Figure 6. Diagnoses in the Trauma Subgroup.

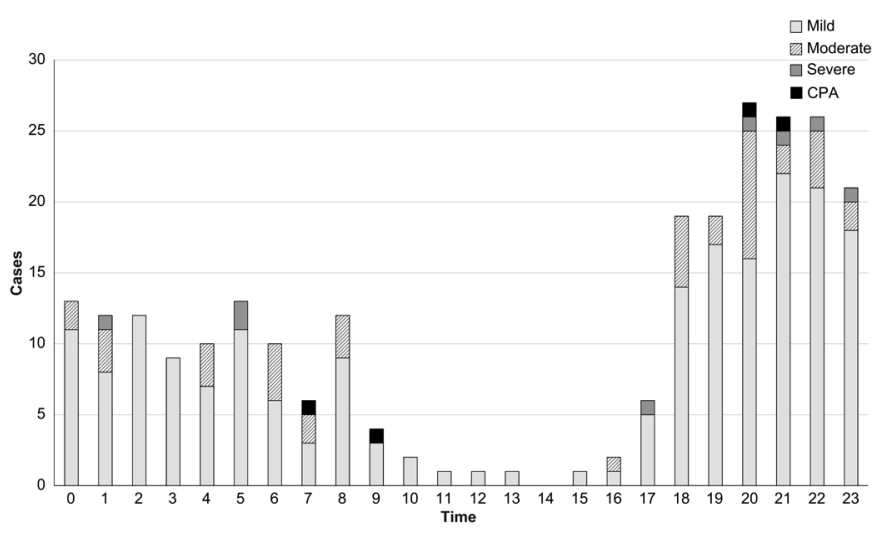

Figure 7. Frequency of Ambulance Dispatch to Hotel/Lodge by Time and Severity.

and Welfare reported that among 5621218 dispatches, $49.3 \%$ of ambulance use was of minor status, $41.0 \%$ was moderate, $8.4 \%$ was severe, $1.4 \%$ was CPA, and $0.1 \%$ was other. ${ }^{8}$

The locality of Mount Fuji is popular for mountain trekking or hiking, so altitude sickness and lower limb injury are common. By contrast, the most common injury on cruise ship travel has been reported to be lower limb fracture, ${ }^{9}$ indicating that injury type reflects the tourist location and typical activities. ${ }^{1}$ July and August reflects the main open period of trekking in Mount Fuji, so it was not surprising that access to emergency services increased at this time.

Most requests originated from hotels/lodge and were typically made in the evening and at nighttime (Figure 7), presumably because of tight travel schedules. Analysis results suggest that there was an increase in the percentage of international travelers requesting ambulances from their hotel or lodge. It is assumed that hotel employees helped guests call the emergency medical services in most instances. However, given that some may have access to a pharmacy, some ambulance requests may have been avoided. Given that Japanese law prohibits the sale of medicine without a pharmacist, this may account for the greater number of ambulance calls in the evening and overnight.

It is assumed that the requests from roads were related to traffic accidents, and not 1 of the 6 CPAs were collected from this location. Traffic accidents are more common in developing countries where there is low compliance with traffic laws, ${ }^{4}$ and driving on different sides of the road is also risky for travelers from countries that drive on the opposite side of the road. ${ }^{10}$ In tropical areas, water and/ or food hygiene can be a problem, but this is not common in Japan. ${ }^{11,12}$ However, animal/bug bites, which are also common in tropical areas, ${ }^{13}$ are a risk in Japan, though the deer, monkey, bear, and boar that are present seldom attack humans. ${ }^{14}$ Rabies is not endemic in Japan. Although mycoses are usually important, ${ }^{15}$ no such cases were seen in this study. Jet lag can occur but was less likely, because most travelers in this study were from Asia. Only one case of deep venous thrombosis was identified in this study.

The current study was limited to a single area, and meaningful generalization of the data will require further research in multiple areas. Another limitation of this study was the failure to include information on the percentage of travelers who required ambulance transport, travel timeframes, preexisting diseases, and outcomes. Some patients might present with a lag time after injury, indicating the potential for data on the time of injury to be inaccurate. Furthermore, the methods of reaching the hospital were unclear (e.g., ambulance, private vehicle, or other).

\section{Conclusion}

Requests for ambulances are increasing among international travelers, with the main reasons being internal disease (53.4\%) and trauma (42.6\%). In this sample, three-quarters of dispatches were for minor complaints, half were from a hotel or lodge, and most were needed outside of normal working hours. 


\section{Research Highlights}

\section{What Is Already Known?}

The main health problems of international travelers are internal diseases, including diarrhea, cough, runny nose, and sore throat.

\section{What This Study Adds?}

Trauma is a major reason for ambulance dispatch accounting for $42.6 \%$ of cases.

\section{Authors' Contributions}

YO was responsible for the study concept, first draft of the manuscript, and the final editing of the manuscript. KT and $\mathrm{KI}$ were responsible for data collection and analysis. $\mathrm{KH}$ and $\mathrm{TN}$ were responsible for data interpretation. NK gave editorial and scientific advice and was the corresponding author.

\section{Conflicts of Interest Disclosures}

The authors declare that they have no conflicts of interest.

\section{Ethical Approval}

This study was approved by the institutional review board of Yamanashi Red Cross Hospital.

\section{Funding/Support}

None.

\section{Acknowledgments}

We thank all the Paramedics of Fujigoko Fire Department for providing the anonymous ambulance dispatch data.

\section{References}

1. Angelo KM, Kozarsky PE, Ryan ET, Chen LH, Sotir MJ. What proportion of international travellers acquire a travel-related illness? a review of the literature. J Travel Med. 2017;24(5). doi:10.1093/jtm/tax046.

2. Olanwijitwong J, Lawpoolsri S, Ponam T, et al. Incidence and spectrum of health problems among travellers to Myanmar. J Travel
Med. 2018;25(1). doi:10.1093/jtm/tax077.

3. Olanwijitwong J, Piyaphanee W, Poovorawan K, et al. Health problems among Thai tourists returning from India. J Travel Med. 2017;24(4). doi:10.1093/jtm/tax013.

4. Piyaphanee W, Kittitrakul C, Lawpoolsri S, et al. Incidence and spectrum of health problems among travelers to Laos. J Travel Med. 2014;21(3):163-168. doi:10.1111/jtm.12107.

5. Long I, Flaherty GT. Traumatic travels-a review of accidental death and injury in international travellers. Int J Travel Med Glob Health. 2018;6(2):48-53. doi:10.15171/ijtmgh.2018.10.

6. Monthly visitor trends report. Japan National Tourism Organization. July, 2019. https://www.jnto.go.jp/jpn/statistics/visitor_trends/. Accessed 13 August 2019.

7. Freedman DO, Chen LH, Kozarsky PE. Medical considerations before international travel. N Engl J Med. 2016;375(3):247-260. doi:10.1056/NEJMra1508815.

8. Current status and issues of emergency medical care system. Ministry of Health, Labour and Welfare. https://www.mhlw.go.jp/ content/10802000/000328610.pdf. Accessed December 17, 2019.

9. Isom WJ, Accilien YD, Chery SB, Mederos-Rodriguez D, Berne JD. Patterns of injury amongst cruise ship passengers requiring hospitalisation. Int Marit Health. 2018;69(4):243-247. doi:10.5603/imh.2018.0039.

10. Stewart BT, Yankson IK, Afukaar F, Medina MC, Cuong PV, Mock C. Road traffic and other unintentional injuries among travelers to developing countries. Med Clin North Am. 2016;100(2):331-343. doi:10.1016/j.mcna.2015.07.011.

11. 11Horneland AM, Jeżewska M, Jaremin B, et al. Establishment of the International Maritime Health Foundation: the next step in scientific publishing in maritime health. Int Marit Health. 2018;69(3):153-156. doi:10.5603/imh.2018.0024.

12. Kuenzli E, Juergensen D, Kling K, et al. Previous exposure in a highrisk area for travellers' diarrhoea within the past year is associated with a significant protective effect for travellers' diarrhoea: a prospective observational cohort study in travellers to South Asia. J Travel Med. 2017;24(5). doi:10.1093/jtm/tax056.

13. Angelo KM, Libman M, Caumes $E$, et al. Malaria after international travel: a GeoSentinel analysis, 2003-2016. Malar J. 2017;16(1):293. doi:10.1186/s12936-017-1936-3.

14. Okano I, Midorikawa Y, Kushima N, et al. Penetrating anorectal injury caused by a wild boar attack: a case report. Wilderness Environ Med. 2018;29(3):375-379. doi:10.1016/j. wem.2018.02.007.

15. Salzer HJF, Stoney RJ, Angelo KM, et al. Epidemiological aspects of travel-related systemic endemic mycoses: a GeoSentinel analysis, 1997-2017. J Travel Med. 2018;25(1). doi:10.1093/jtm/tay055. 